\title{
Exploración física del abdomen agudo y sus principales signos como una práctica basada en la evidencia
}

\author{
Physical exam of acute abdomen \\ and its main signs as an evidence-based practice \\ David R de Rungs Brown,* André Víctor Baldin,* \\ J Muñoz Hinojosa,* A Valdés Castañeda, ${ }^{*}$ M Gómez Palacio*
}

Palabras clave: Medicina basada en la evidencia, exploración física, diagnóstico, semiología, prueba científica.

Key words: Evidence-based medicine, physical examination, diagnosis, semiology, scientific proof.

* Departamento de Cirugía General del Hospital Ángeles Lomas.

Recibido: 01/01/2015 Aceptado: 01/05/2015

\section{RESUMEN}

\section{ABSTRACT}

Introducción: La medicina basada en la evidencia combina la mejor prueba científica disponible junto con el juicio clínico para tomar decisiones médicas. El retorno al ritual de la exploración física y actualizarlo a la medicina basada en evidencia son de gran importancia en esta etapa de la medicina tecnológica. Material y métodos: Se realizó un estudio comparativo de tipo metaanálisis de investigación educativa utilizando ocho bases de datos, buscando artículos de los últimos 30 años (1982-2012). De 50 artículos seleccionados, se pusieron a prueba para la homogeneidad de los datos mediante el uso de la estadística de prueba odds ratio de Breslow-Day. Los estudios se combinaron y los valores predictivos positivo y negativo fueron calculados mediante la prueba Cochran-Mantel-Haenszel. Resultados: Las tres principales causas de dolor abdominal agudo en el Servicio de Urgencias $(n=12,706)$ fueron apendicitis, colecistitis y oclusión intestinal, incluyendo los principales signos encontrados en abdomen agudo con alguna patología crónica digestiva. Apendicitis $(\mathrm{n}=9,478)$ : dolor abdominal y el signo de Von Blumberg, valor predictivo positivo (VPP) de 7.9 (7.3 a 8.5), punto de McBurney con VPP de 4.2 (1.16.3). Colecistitis $(\mathrm{n}=5,716)$ : signo Murphy positivo con VPP de 2.8 (0.8-8.6), rigidez abdominal con VPP de 1.6 (1.0-2.5). Oclusión intestinal ( $\mathrm{n}=2,275)$ : peristalsis visible con VPP de 9.5 (16.3 a 22.4) y distensión abdominal con VPP de 6.3 (09.03 a 12.03). Exploración abdominal para hepatopatía crónica $(\mathrm{n}=3,134)$ : signo de ola para encontrar ascitis libres con VPP 6 (3.3 a 11.0), la palpación para la búsqueda de esplenomegalia con el paciente en decúbito supino con VPP de 8.2 (5.8-12) y hepatomegalia palpable en el borde hepático con VPP de 2 (1.5-2.8). Conclusión: Desde el inicio de la semiología, para llegar a un diagnóstico pertinente, la exploración física siempre es fundamental. Las tres principales patologías pueden tener su diagnóstico presuntivo realizando el examen adecuado del paciente, lo que demuestra que la medicina basada en la evidencia y el ritual de la exploración física es el mejor método diagnóstico.
Introduction: Evidence-based medicine combines the best scientific evidence available and clinical judgment in order to make medical decisions. The return to the ritual of the physical examination and its upgrading to evidencebased medicine are important in this age of technological medicine. Methods: A comparative meta-analysis was performed using eight databases, searching for articles in the past 50 years. A total of 90 articles were selected, which were then tested for homogeneity of data by using the odds ratio Breslow-Day statistic test. The studies found were combined and positive and negative predictive values were calculated using the Cochran-Mantel-Haenszel test. Results: The three main causes of acute abdominal pain in the emergency department ( $n=12,706)$, were determined as appendicitis, cholecystitis and intestinal occlusion, including the primary signs found in acute abdomen with a chronic digestive disease. Appendicitis $(n=9,478)$ : right lower quadrant pain with a positive predictive value (PPV) of 7.9 (7.3-8.5), and Von Blumberg sign, McBurney's point with a PPV of 4.2 (1.1-6.3). Cholecystitis $(n=5,716)$ : positive Murphy sign with a PPV of 2.8 (0.8-8.6), and abdominal rigidity with a PPV of 1.6 (1.0-2.5). Intestinal occlusion ( $n=2,275)$ : visible peristalsis with a PPV of 9.5 (16.3-22.4), and abdominal distension with a PPV of 6.3 (9.3-12.3). Abdominal exploration in chronic liver diseases ( $n=3,134)$ : wave sign for finding free ascites with a PPV of 6 (3.3-11.0), palpation with the patient supine in the search of a splenomegaly with a PPV of 8.2 (5.8-12), and search for palpable hepatomegaly in the liver edge with a PPV of 2(1.5-2.8). Conclusion: Physical examination has always been essential to reach an appropriate diagnosis since the beginning of semiotics. The three main pathologies detected in this study can have their presumptive diagnosis through an appropriate examination of the patient, demonstrating that evidence-based medicine and the ritual of the physical examination are the best diagnostic examination method. 


\section{INTRODUCCIÓN}

$\mathrm{L}$ a medicina basada en evidencia (MBE) combina la mejor prueba científica disponible para un juicio clínico. ${ }^{1-3}$ Los signos y síntomas del paciente son cruciales para la toma de decisiones médicas. ${ }^{4,5}$ La MBE es una codificación y modernización de las bases de la ciencia, la medicina y la probabilidad, integrando un diagnóstico presuntivo y específico para el paciente. ${ }^{6,7} \mathrm{El}$ conocimiento y las destrezas necesarias para la aplicación crítica de la literatura y la práctica de la MBE usualmente se enseñan en cursos y talleres en los salones de clase de las facultades de medicina, los cuales no tienen contacto con la práctica clínica. ${ }^{8-10}$

En años recientes, se ha dado una importancia extraordinaria a los estudios de imagen y laboratorio, dejando de lado la clínica ortodoxa y la relación médico-paciente; el retomar el "ritual de la exploración física" y actualizarlo a la MBE es de esencial importancia en esta era de la medicina tecnológica. ${ }^{11,12}$ La MBE es una secuencia de una serie de procesos que integran una forma de actuar y pensar, $y$ un estilo de práctica. ${ }^{13,14}$ Consiste en la investigación eficaz de la información con base en un problema o pregunta real, la evaluación mediante habilidades de lectura crítica de la literatura médica y la aplicación de lo que se encuentre en esta búsqueda a la toma de decisiones para una recomendación informada entre profesionales de la salud, gestores de salud y pacientes. ${ }^{15,16}$

Se considera que la MBE está integrada por tres componentes que van a permitir que se tomen mejores decisiones clínicas respecto al cuidado de la salud del individuo: ${ }^{17}$ primero, el nivel de evidencia de la literatura científica; segundo, la expresión de la sintomatología del paciente $y$, tercero, la experiencia del profesional de salud; éstas son las variables que, al combinarse con el contexto institucional, pueden jerarquizar los criterios del conocimiento reportados en el área de la salud para obtener un mejor resultado. ${ }^{18,19}$

El propósito de este trabajo es retomar la importancia de la clínica ortodoxa actualizada a la MBE y aportar a la ciencia médica la trascendencia de aprender una adecuada interpretación de los signos clínicos para el diagnóstico en abdomen agudo, además de determinar los signos que aporten mayor evidencia clínica para el diagnóstico o exclusión de las tres principales causas de dolor abdominal agudo, así como las maniobras con mayor sensibilidad para la búsqueda de ascitis, esplenomegalia y hepatomegalia como signos de exploración más frecuentes en abdomen agudo en enfermedades digestivas crónicas. ${ }^{20,21}$

\section{MATERIAL Y MÉTODOS}

Se realizó un estudio comparativo de tipo metaanálisis de investigación educativa utilizando ocho bases de datos, buscando artículos de los últimos 30 años (1982-2012). De 50 artículos seleccionados, se crearon tablas de $2 \times 2$, y posteriormente fueron probadas para homogeneidad de datos mediante la razón de momios utilizando la prueba estadística Breslow-Day. $\mathrm{Si}$ los estudios no fueron rechazados como heterogéneos por dicha prueba $(p=0.05)$, los intervalos de confianza fueron revisados manualmente para excluir errores tipo II. Los estudios que cumplieron ambos criterios fueron combinados y se calcularon los valores predictivos positivo y negativo mediante el método de Cochran-Mantel-Haenszel utilizando el programa Statistical Package for the Social Sciences (SPSS, versión 16.0, Chicago. IL, EUA). Se determinaron los signos y síntomas con mayor sensibilidad y especificidad para las tres principales causas de dolor abdominal agudo: apendicitis, colecistitis y oclusión intestinal; y las maniobras orientadas hacia la búsqueda de ascitis, esplenomegalia y hepatomegalia como signos de exploración más frecuentes en abdomen agudo en enfermedades digestivas crónicas. Finalmente, se obtuvieron gráficas de Forest utilizando el programa Metadata Viewer (versión 1.04, Atlanta, GA, EUA).

\section{RESULTADOS}

Se definieron las tres principales causas de ingreso por dolor abdominal agudo en el Departamento de Urgencias de cada estudio (n $=20,603$ ), excluyendo a 7,897 pacientes que no cumplieron con los criterios de inclusión mencionados, obteniendo un total real de $\mathrm{n}=$ 12,706 con apendicitis, colecistitis y oclusión intestinal, así como las causas de abdomen agudo en enfermedades digestivas crónicas. 
Figura 1.

Signos de apendicitis con mayor valor predictivo positivo (VPP). Se muestran los rangos inferior $y$ superior, así como la media estimada en círculos. La línea roja representa el VPP = 1 y la línea azul VPP = 4. Intervalo de confianza $(I C)=95 \%$
- Apendicitis ( $n=9,478$ ). Se definieron los seis principales signos que aportan mayor valor predictivo positivo (VPP), y de ellos, los dos principales son dolor en cuadrante inferior derecho, con VPP de 7.9 (7.3-8.5), y signo de Von Blumberg (rebote) positivo en punto de McBurney, con VPP de 4.2 (1.1-6.3). Signo de psoas positivo con VPP 3.4 (2.3-5.2); dolor migratorio con VPP 4.0 (4.5-6.3); rigidez abdominal con VPP de 5.5 (4.9-7.2); anorexia con VPP de 1.0 (1.2-2.1) (Figura 1).

- Colecistitis ( $n=5,716$ ). Se identificaron los cuatro principales signos que aportan mayor valor predictivo positivo (VPP), y de ellos, los dos principales son signo de Murphy positivo, con VPP de 2.8 (0.88.6), y rigidez abdominal, con VPP de 1.6 (1.0-2.5). Asimismo, se determinó el VPP para la emesis con 1.5 (1-3.2); dolor en hipocondrio derecho con VPP 0.9 (1-3.5); rebote con VPP 0.7 (1.0-3.6); con los signos presentados se puede aportar evidencia de su futilidad clínica (Figura 2).

- Oclusión intestinal ( $n=2,275)$. Se determinaron los seis principales signos que aportan mayor VPP; de ellos, los dos principales son peristalsis visible, con VPP de 9.5 (16.322.4), y distensión abdominal, con VPP de 6.3 (9.3-12.3). Los signos menos sugestivos fueron peristalsis proximal aumentada, con VPP 7.1 (5.3-8.2) y rigidez abdominal, con VPP de 0.5 (0.1-2.1) (Figura 3).

- Maniobras de exploración abdominal para enfermedades digestivas crónicas (n $=3,134$ ). Se determinaron dos maniobras de exploración para la búsqueda de cada una de las siguientes alteraciones: ascitis, esplenomegalia y hepatomegalia. Para cada una de las alteraciones, la maniobra que aporta mayor VPP son: signo de la ola para la búsqueda de ascitis libre, con VPP de 6 (3.3-11.0); matidez a la percusión, con VPP de 3.3 (2.0-5.0); la palpación a una mano con el paciente en decúbito supino para la búsqueda de esplenomegalia, con VPP de 8.2 (5.8-12), y el borde hepático palpable para la búsqueda de hepatomegalia con VPP de 2 (1.5-2.8) (Figura 4).

\section{DISCUSIÓN}

La exploración física ortodoxa debe continuarse como un método de recolección de informa-

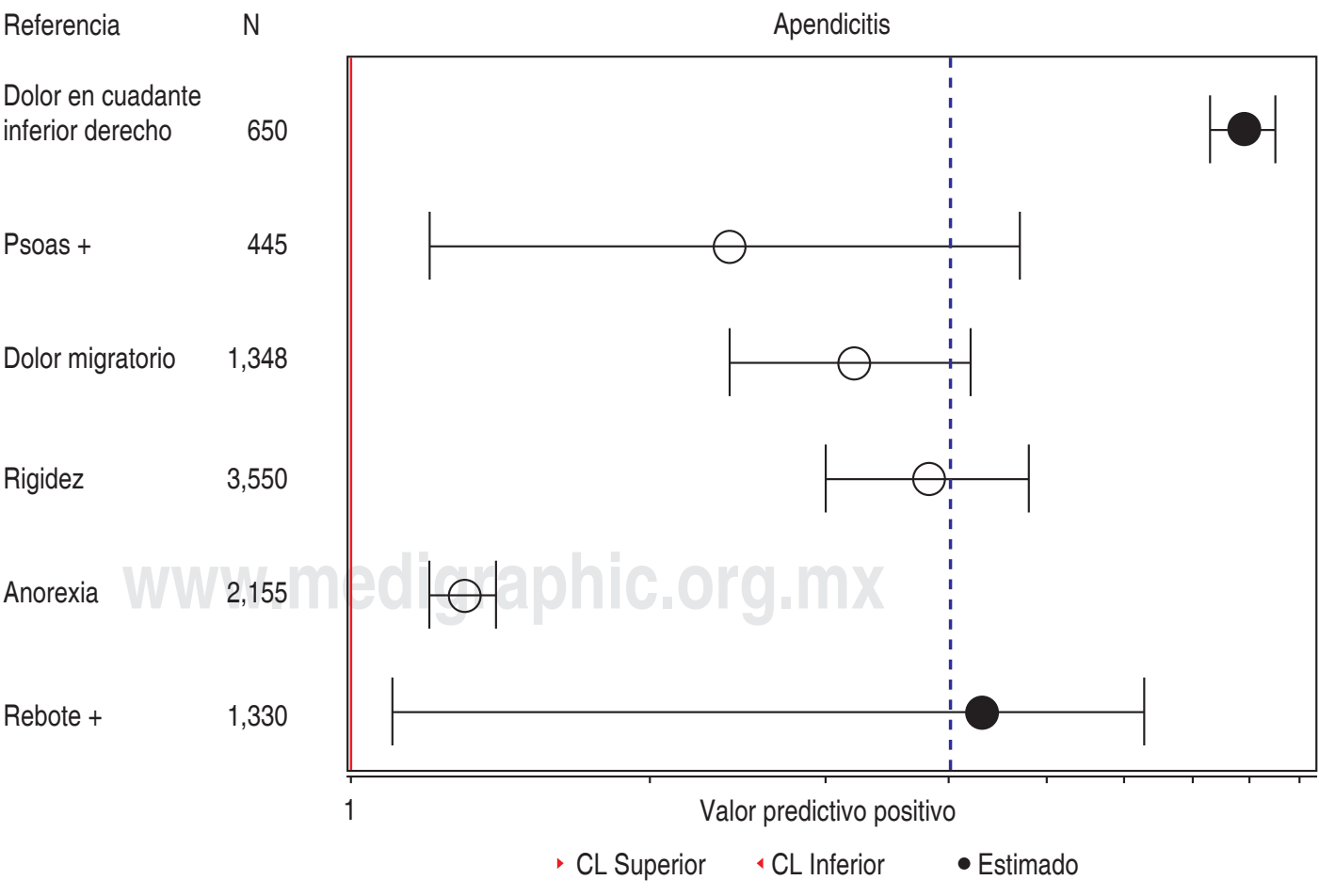




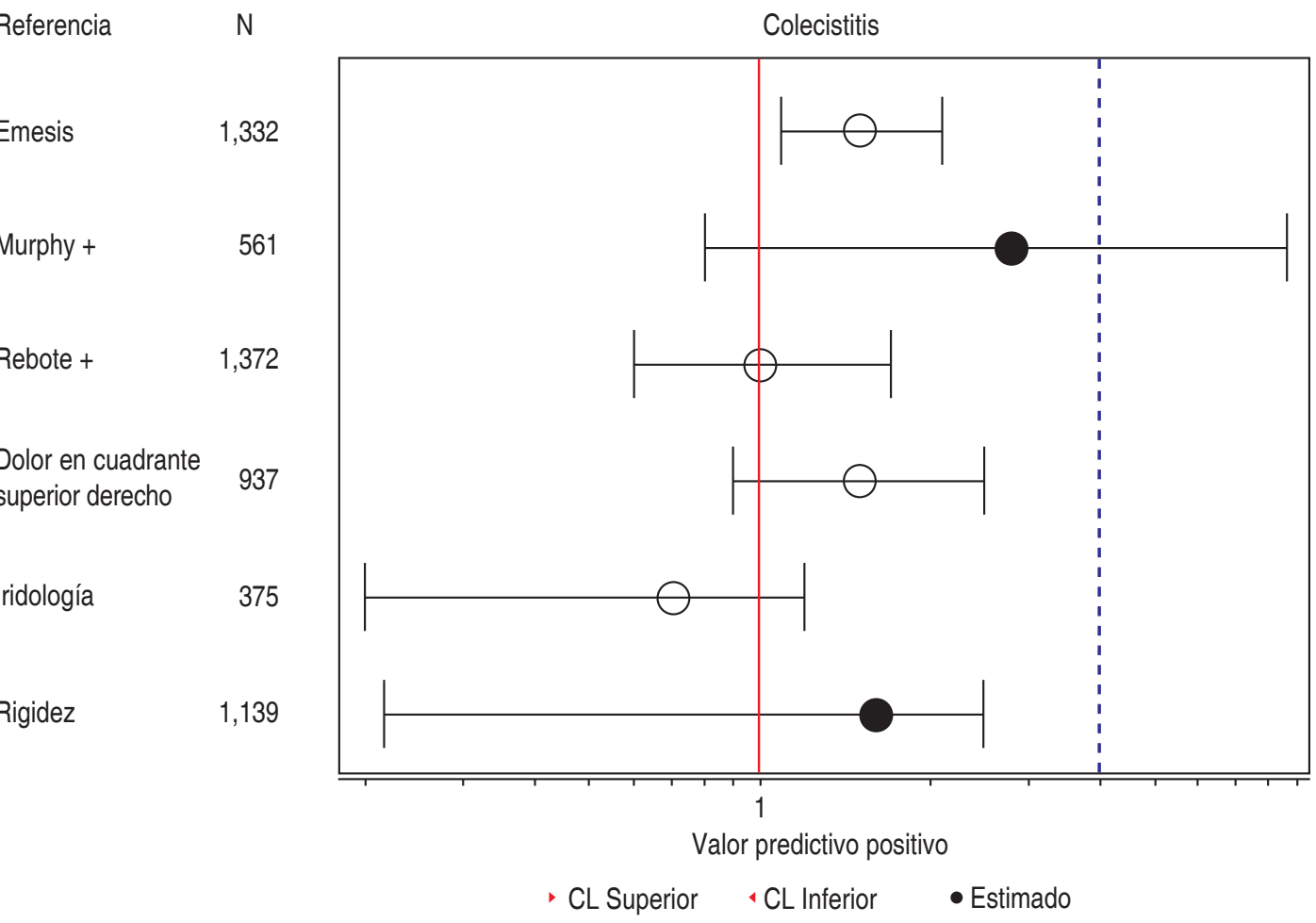

Figura 2.

Signos de colecistitis con mayor valor predictivo positivo (VPP). Se muestran los rangos inferior y superior, así como la media estimada en círculos. La línea roja representa VPP = 1 y la línea azul $V P P=4$. Intervalo de confianza $(I C)=$ 95\%.

\section{Figura 3.}

Signos de oclusión intestinal con mayor valor predictivo positivo (VPP). Se muestran los rangos inferior y superior, así como la media estimada en círculos. La línea roja representa VPP = 1 y la línea azul VPP $=4$. Intervalo de confian$z a(I C)=95 \%$.

Referencia

Peristalsis visible

Distensión abdominal

Peristalsis aumentada

Peristalsis abolida

Rigidez

Rebote +

N 455 623

355 298

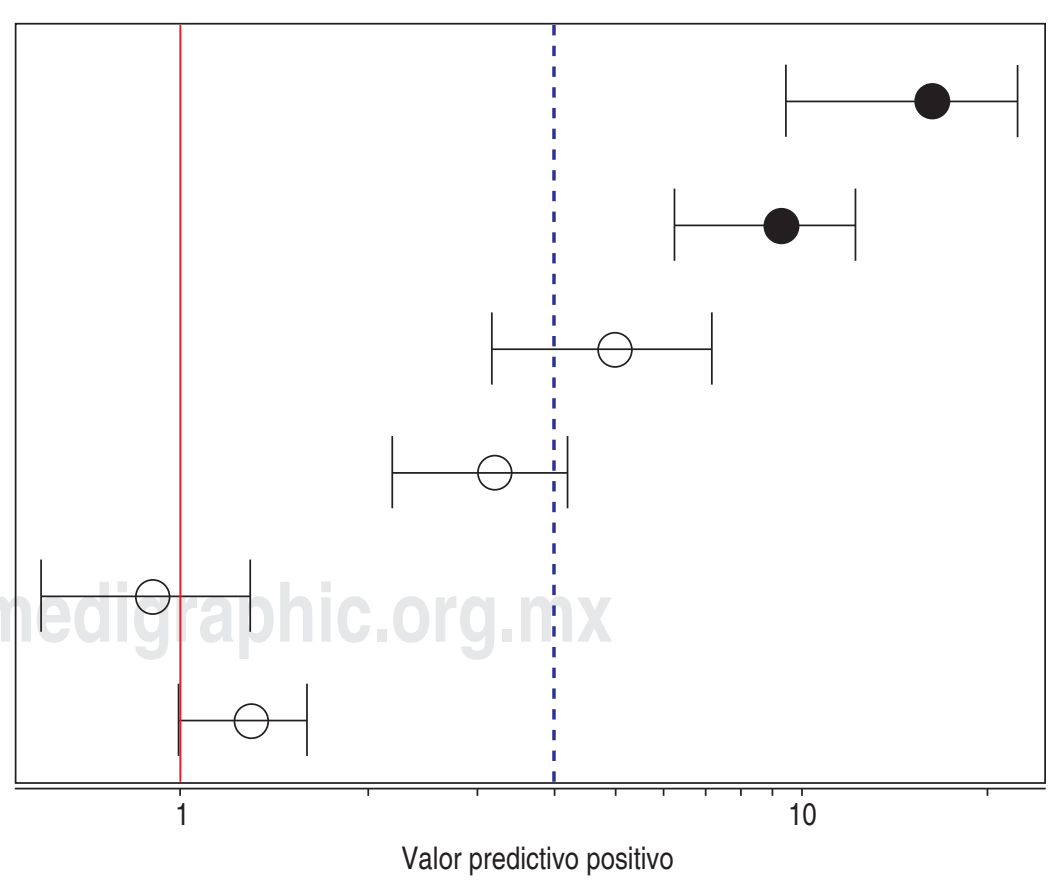

- CL Superior ‘ CL Inferior • Estimado 
Fig. 4.

Maniobras encaminadas a la búsqueda de ascitis (A), esplenomegalia (E) y hepatomegalia $(\mathrm{H})$. Se muestran los rangos inferior $y$ superior, así como la media estimada en círculos. La línea roja representa valor predictivo positivo (VPP) $=1$ y la línea azul VPP $=4$. Intervalo de confianza (IC) = 95\%.
Referencia

Matidez camb.

Signo de la ola

Percusión técnica

Nixon

Palpación a una mano

Prueba de rascado

Borde palpable

$$
\text { N Tipo }
$$

Exploración abdominal

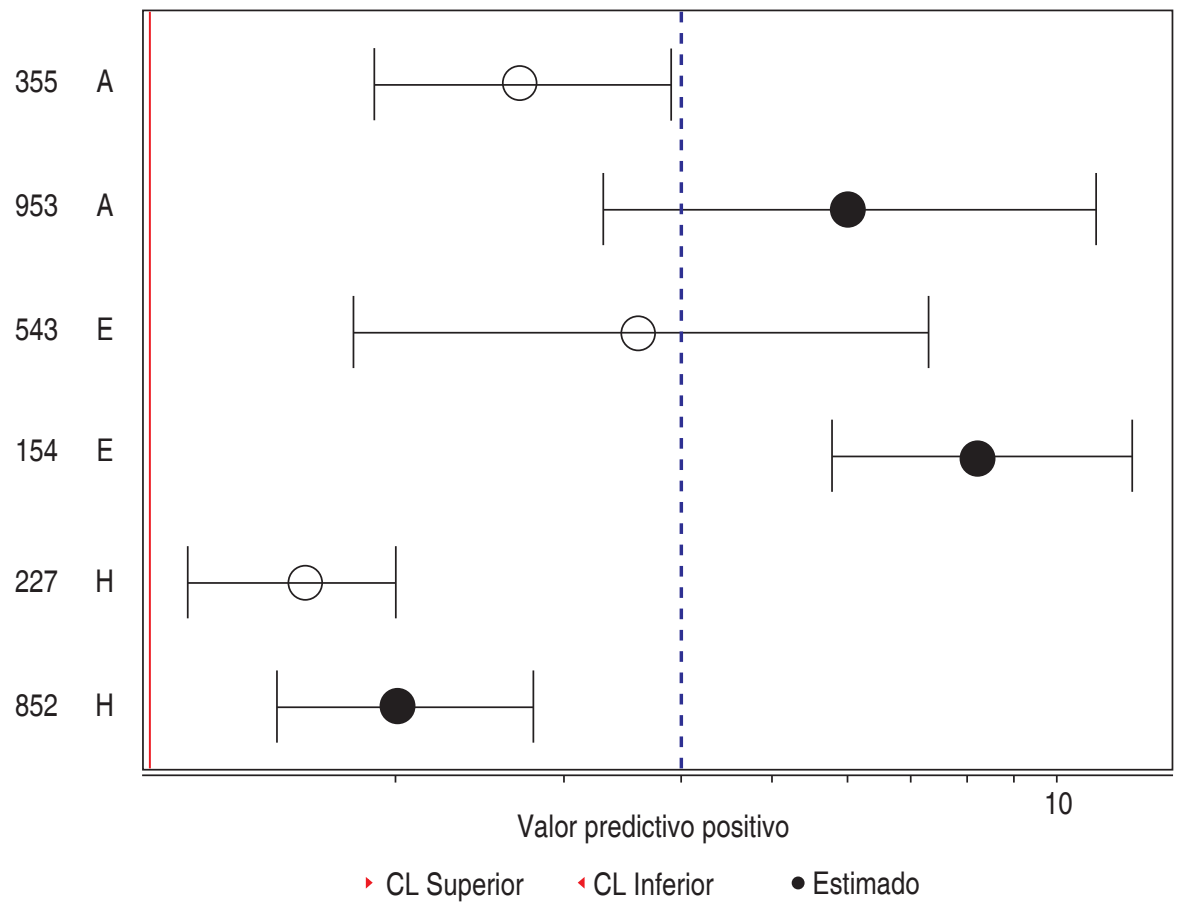

ción para obtener una semiología y llegar al diagnóstico presuntivo. Se ofrecen los signos y síntomas con mayor VPP para las tres principales causas de dolor abdominal agudo, así como algunas maniobras de exploración física con VPP altos. La evidencia que se aporta mediante el presente metaanálisis ofrece evidencia sólida, ya que todas las pruebas estadísticas son paramétricas y mediante intervalos de confianza, y no por medio de prueba de hipótesis. La exploración física ortodoxa puede llevarse a la MBE y competir con pruebas de imagen o laboratorio para el diagnóstico de estas enfermedades que presentan abdomen agudo en algún momento de su evolución.

\section{CONCLUSIÓN}

En nuestro afán cotidiano hacia una medicina cada día más científica y tecnológica hay algo que parece que estamos pasando por alto, un factor al que usualmente le restamos importancia y que prevalece por mucho que progresen los procedimientos actuales para reconocer y tratar la enfermedad. No debemos olvidar que por encima de toda esa gama desbordante de sistemas e instrumentos existirán siempre dos constantes inmutables: el médico y el enfermo. Antes que la tecnología — que podremos usar sólo de soporte dudoso—, la exploración física es un método muy asertivo para el diagnóstico.

Hoy más que nunca, tenemos que integrar la medicina clínica como el componente básico de nuestra profesión ante la medicina que actualmente se sustenta y respalda con métodos diagnósticos de altos costos para el paciente.

\section{REFERENCIAS}

1. Purcell TB. Nonsurgical and extraperitoneal causes of abdominal pain. Emerg Med Clin North Am. 1989; 7: 721-740.

2. Brewer BJ, Golden GT, Hitch DC, Rudolf LE, Wangensteen SL. Abdominal pain. An analysis of 1,000 consecutive cases in a University Hospital emergency room. Am J Surg. 1976; 131: 219-223.

3. Cummings S, Papadakis M, Melnick J, Gooding GA, Tierney LM, Jr. The predictive value of physical examinations for ascites. West J Med. 1985; 142: 633-636.

4. Gerspacher-Lara R, Pinto-Silva RA, Serufo JC, Rayes AA, Drummond SC, Lambertucci JR. Splenic palpation for the evaluation of morbidity due to schistosomiasis mansoni. Mem Inst Oswaldo Cruz. 1998; 93: 245-248.

5. Joshi R, Singh A, Jajoo N, Pai M, Kalantri SP. Accuracy and reliability of palpation and percussion for detecting hepatomegaly: a rural hospital-based study. Indian J Gastroenterol. 2004; 23: 171-174. 
6. Berry J Jr, Malt RA. Appendicitis near its centenary. Ann Surg. 1984; 200: 567-575.

7. Nauta RJ, Magnant C. Observation versus operation for abdominal pain in the right lower quadrant. Roles of the clinical examination and the leukocyte count. Am J Surg. 1986; 151: 746-748.

8. Izbicki JR, Knoefel WT, Wilker DK, Mandelkow HK, Muller K, Siebeck M, et al. Accurate diagnosis of acute appendicitis: a retrospective and prospective analysis of 686 patients. Eur J Surg. 1992; 158: 227-231.

9. Alvarado A. A practical score for the early diagnosis of acute appendicitis. Ann Emerg Med. 1986; 15: 557-564.

10. Fenyö G. Routine use of a scoring system for decisionmaking in suspected acute appendicitis in adults. Acta Chir Scand. 1987; 153: 545-551.

11. Pocock SJ. The historical development of clinical trials. Chapter 2. In: Clinical Trials. Toronto: John Wiley \& Sons; 1984 . pp. 14-15

12. Daniels M, Hill AB. Chemotherapy of pulmonary tuberculosis in young adults. BMJ. 1952; I: 1162-1168.

13. Valdivieso V. La medicina basada en evidencias. Rev Med Chile. 1997; 125: 1103-1109.

14. Guyatt G. Users' guides to the medical literature: a manual for evidence-based clinical practice. Eds. G Guyatt, D Rennie, 2002. p. xiv.

15. Evidence Based Medicine Working Group. Evidence Based Medicine. A new approach to teaching the practice of medicine. JAMA. 1992; 268: 2420-2425.

16. Verdugo RJ, Salinas RS, Castillo J, Cea JG. Surgical versus non surgical treatment for carpal tunnel syndrome (Cochrane Review). In: The Cochrane Library. 2003; 2.
17. Counsell C, Salinas R, Warlow C, Naylor R. Patch angioplasty versus primary closure for carotid endarterectomy (Cochrane Review). In: The Cochrane Library. 2003; 2 .

18. Rojas MP, Telaro E, Russo A, Fossati R, Palli D, Rosselli Del Turco $M$ et al. Follow-up strategies for women treated for early breast cancer (Cochrane Review). In: The Cochrane Library. 2003; 2.

19. Salinas RA, Alvarez G, Alvarez MI, Ferreira J. Corticosteroids for Bell's palsy (idiopathic facial paralysis) (Cochrane Review). In: The Cochrane Library. 2003; 2.

20. Salinas R, Counsell C, Prasad K, Gelband H, Garner P. Ministry of Health, Santiago, Chile. Treating neurocysticercosis medically: a systematic review of randomized, controlled trials. Trop Med Int Health. 1999; 4: 713-718.

21. Letelier LM, Udol K, Ena J, Weaver B, Guyatt G. Effectiveness of amiodarone for conversion of atrial fibrillation to sinus rhythm: a meta-analysis. Arch Intern Med. 2003; 163: 777-785.

Correspondencia:

\section{Dr. David R. de Rungs Brown}

Bosque de Bugambilias Núm. 1000, A-401

Col. Bosques de las Lomas

Del. Cuajimalpa, 05120, México, DF.

E-mail: De_rungs@hotmail.com 